Noname manuscript No.

(will be inserted by the editor)

\title{
Organic Compounds in Circumstellar and Interstellar Environments
}

\section{Sun Kwok}

Received: date / Accepted: date

\begin{abstract}
Recent research has discovered that complex organic matter is prevalent throughout the Universe. In the Solar System, it is found in meteorites, comets, interplanetary dust particles, and planetary satellites. Spectroscopic signatures of organics with aromatic/aliphatic structures are also found in stellar ejecta, diffuse interstellar medium, and external galaxies.

From space infrared spectroscopic observations, we have found that complex organics can be synthesized in the late stages of stellar evolution. Shortly after the nuclear synthesis of the element carbon, organic gas-phase molecules are formed in the stellar winds, which later condense into solid organic particles. This organic synthesis occurs over very short time scales of about a thousand years.

In order to determine the chemical structures of these stellar organics, comparisons are made with particles produced in the laboratory. Using the technique of chemical vapor deposition, artificial organic particles have been created by injecting energy into gas-phase hydrocarbon molecules. These comparisons led us to believe that the stellar organics are best described as amorphous carbonaceous nanoparticles with mixed aromatic and aliphatic components.
\end{abstract}

The chemical structures of the stellar organics show strong similarity to the insoluble organic matter found in meteorites. Isotopic analysis of meteorites and interplanetary dust collected in the upper atmospheres have revealed the presence of pre-solar grains similar to those formed in old stars. This provides a direct link between star dust and the Solar System and raises the possibility

S. Kwok

Space Astronomy Laboratory, Faculty of Science, The University of Hong Kong, Hong Kong, China

Tel.: +852-3917-2682

Fax: +852-2858-4620

E-mail: sunkwok@hku.hk 
that the early Solar System was chemically enriched by stellar ejecta with the potential of influencing the origin of life on Earth.

Keywords organic matter · stellar evolution · origin of life

\section{Introduction}

Baryonic matter in the Universe can be in several forms. The ionized (plasma) component was the first state of matter to be studied. It manifests itself in the form of self-luminous gaseous bodies such as stars, gaseous nebulae, and collections of stars (star clusters, galaxies). The molecular state of matter was discovered in the 20th century and over 170 interstellar molecules have been detected through their rotational and vibration transitions using the techniques of millimeter-wave and infrared spectroscopy, respectively. The solid state of matter in the form of interstellar grains was first studied through selective extinction in the optical and later through self-emission in the infrared.

Early models based on the shape of the extinction curve generally assumed graphite, iron, or ice as the chemical ingredients of interstellar solid grains. With advances of infrared spectroscopy, it is now possible to identify the chemical contents of interstellar grains through comparisons with laboratory spectroscopy. Crystalline solids have highly ordered lattice structures and have optically active lattice vibrational modes and a number of minerals have been identified in the interstellar medium in this way. In comparison, organic amorphous solids have many electronic states resulting in broad absorption bands. If macromolecular organic solids solids exist in space, they can be identified by their visible colors, albedo, absorption bands in the visible/UV, and infrared virbational bands in either emission or absorption. In this paper, we discuss the nature of organic solids in stellar and interstellar environments and their possible relationship with organic matter found in the Solar System.

\section{Stellar synthesis of organics}

The element carbon $(\mathrm{C})$ is synthesized during the late stages of stellar evolution in the asymptotic giant branch (AGB) phase. The element is dredged up from the core to the surface through convective processes in the stellar envelope. Near the end of the AGB, a strong stellar wind develops which in less than one million years can completely deplete the stellar envelope. Molecules begin to form under low temperature and density conditions in the outflow. As the result of such mass loss, circumstellar envelopes are built up around AGB stars and over 70 circumstellar molecules have been detected through their rotational transitions. The kinds of molecules detected include inorganics $(\mathrm{CO}, \mathrm{SiO}, \mathrm{SiS})$, organics $\left(\mathrm{C}_{2} \mathrm{H}_{2}, \mathrm{CH}_{4}, \mathrm{C}_{2} \mathrm{H}, \mathrm{C}_{4} \mathrm{H}, \mathrm{C}_{5}, \mathrm{H}_{2} \mathrm{CO}\right)$, nitrogen species ( $\mathrm{HCN}, \mathrm{NH}_{3}, \mathrm{C}_{3} \mathrm{~N}, \mathrm{CH}_{3} \mathrm{CN}$ ), phosphorus compounds $\left(\mathrm{CP}, \mathrm{PH}_{3}, \mathrm{PN}\right.$ ), radicals $\left(\mathrm{CN}, \mathrm{C}_{2} \mathrm{H}, \mathrm{C}_{3}, \mathrm{HCO}^{+}\right)$, metals $(\mathrm{AlCl}, \mathrm{MgNC}, \mathrm{ALF}, \mathrm{NaCN}, \mathrm{KCl}$ ), 
rings $\left(\mathrm{C}_{3} \mathrm{H}_{2}\right)$, and chains $\left(\mathrm{HC}_{3} \mathrm{~N}, \mathrm{HC}_{5} \mathrm{~N}, \mathrm{HC}_{7} \mathrm{~N}\right)$ (Cernicharo et al. 2011; Ziurys 2014). AGB stars are prolific molecular factories.

As soon as the outflow reaches certain distances from the star such that the temperature drops below the condensation temperature, solids condense from the gas phase in the stellar winds. The most common kinds of solids detected are amorphous silicates and silicon carbide (SiC) (Kwok et al. 1997). These solids manifest themselves through infrared emissions due to lattice vibrational stretching and bending modes. In fact, the amount of solids produced is so large that some stars can be completely obscured by circumstellar materials and the optical output of star light is totally converted to infrared continuum emission by circumstellar grains. A strong excess of infrared radiation is therefore an observational characteristics of evolved stars.

When the stellar wind completely depletes the stellar envelope and the core is exposed, ultraviolet radiation from the hot core can photoionize the cicrumstellar gas, resulting in the illumination of the circumstellar nebula through the emission of the recombination lines of hydrogen and helium and collisionally excited lines of heavy elements. These bright visible objects are called planetary nebulae (Kwok 2000). One of the most unexpected discoveries in 20th century astronomy was the detection of the unidentified infrared emission (UIE) features in the spectrum of planetary nebulae. A family of broad infrared bands at 3.3, 6.2, 7.7, 8.6 and $11.3 \mu \mathrm{m}$ was found in the spectrum of the planetary nebulae NGC 7027 (Russell et al. 1977). These UIE bands are later found to be common in C-rich planetary nebulae, interstellar emission and reflection nebulae, diffuse clouds in the galactic halo, and external galaxies. The strengths of the UIE bands are so strong that up of $20 \%$ of the total energy output from some galaxies is emitted in these bands (Smith et al. 2007).

In order to determine the origin of these UIE bands, it would be useful to first find out when their carrier is first synthesized. While the UIE bands are strong in planetary nebulae, they are not seen in their progenitor AGB stars. The carrier of the UIE bands must therefore be synthesized during the evolutionary stage between the AGB and planetary nebulae stages. Objects in this transition stage are called pre- or proto-planetary nebulae. These transition objects are difficult to identify in the optical because their circumstellar materials are neutral and do not have emission lines. The first identification of pre-planetary nebulae was therefore achieved through their infrared properties (Kwok 1993). Follow-up infrared spectroscopic studies of these objects show that they already possess UIE bands. This suggests that the carriers of the UIE bands are formed during the pre-planetary nebulae stage (Kwok et al. 1999). Since the transition from AGB to planetary nebulae only takes a few thousand years, we can conclude that the carrier of UIE bands are synthesized over similar time scales. 


\section{Carrier of the unidentified infrared emission bands}

Soon after the discovery, the UIE bands were identified as stretching and bending modes of aromatic compounds (Knacke 1977; Duley et al. 1979, 1981; Puetter et al. 1979). The resemblance of the UIE bands to the spectrum of automobile exhaust led to the suggestion that the carrier of the UIE bands are polycyclic aromatic hydrocarbon (PAH) molecules (Allamandola et al. 1985). Since PAH molecules are simple, the PAH hypothesis has become the most popular explanation to the UIE phenomenon in the astronomical community. Although there have been a number of alternative models proposed: including hydrogenated amorphous carbon (Jones et al. 1990), quenched carbonaceous composites (Sakata et al. 1987), coal and kerogen (Papoular et al. 1989; Papoular 2001), or petroleum fractions (Cataldo et al. 2004), they were never able to challenge the dominant position of the $\mathrm{PAH}$ hypothesis.

The detection of aliphatic features at 3.4 and $6.9 \mu \mathrm{m}$ in pre-planetary nebulae and planetary nebulae raised doubts about the carrier of the UIE bands being a pure aromatic species like PAH. The presence of strong and broad plateau emission features around 8 and $12 \mu \mathrm{m}$ also suggests the existence of aliphatic side groups (Kwok et al. 2001). The wide presence of the 3.4 $\mu \mathrm{m}$ feature in absorption in the Galaxy (Wickramasinghe and Allen 1980; Pendleton et al. 1994) and external galaxies (Spoon et al. 2004; Dartois et al. 2007; Yamagishi et al. 2012) has raised our consciousness about the importance of the aliphatic component. In fact, the strengths of the aliphatic features are such that at at least $15 \%$ of the carbon reservoir in our Galaxy must be in the form of $s p^{3}$ bonded (Dartois 2011).

These developments led to the proposal that the carriers of UIE are mixed aromatic/aliphatic organic nanoparticles (MAON, Kwok and Zhang 2011, 2013). MAONs are 3-dimensional structures consisting of aromatic rings of different sizes and aliphatic chains of different lengths and random orientations. They also contain impurities such as $\mathrm{O}, \mathrm{S}$, and $\mathrm{N}$. The exact aromatic to aliphatic ratio depends on the radiation environment, the original gas-phase components, and the $\mathrm{H}$ content.

\section{Chemical structure of interstellar organics}

Astronomical observations have shown that the interstellar medium of our Galaxy and external galaxies are filled with complex organics. These organics have both aromatic and aliphatic components and may not have a fixed structure. It is well known that carbonaceous solids with mixed hybridization states are natural products of combustion. Soot is the result of combustion of hydrocarbon molecules in a flame and has a chemical structure that consists of islands of aromatic rings connected by aliphatic chains (Chung and Violi 2011). The side groups can be made of $\mathrm{C}$ and $\mathrm{H}\left(-\mathrm{CH}_{3},-\mathrm{CH}_{2}\right)$, or include heavy elements such as $\mathrm{O}$ and $\left.\mathrm{N}(-\mathrm{HCO}),-\mathrm{OH},-\mathrm{NH}_{2}\right)$. These heavy 


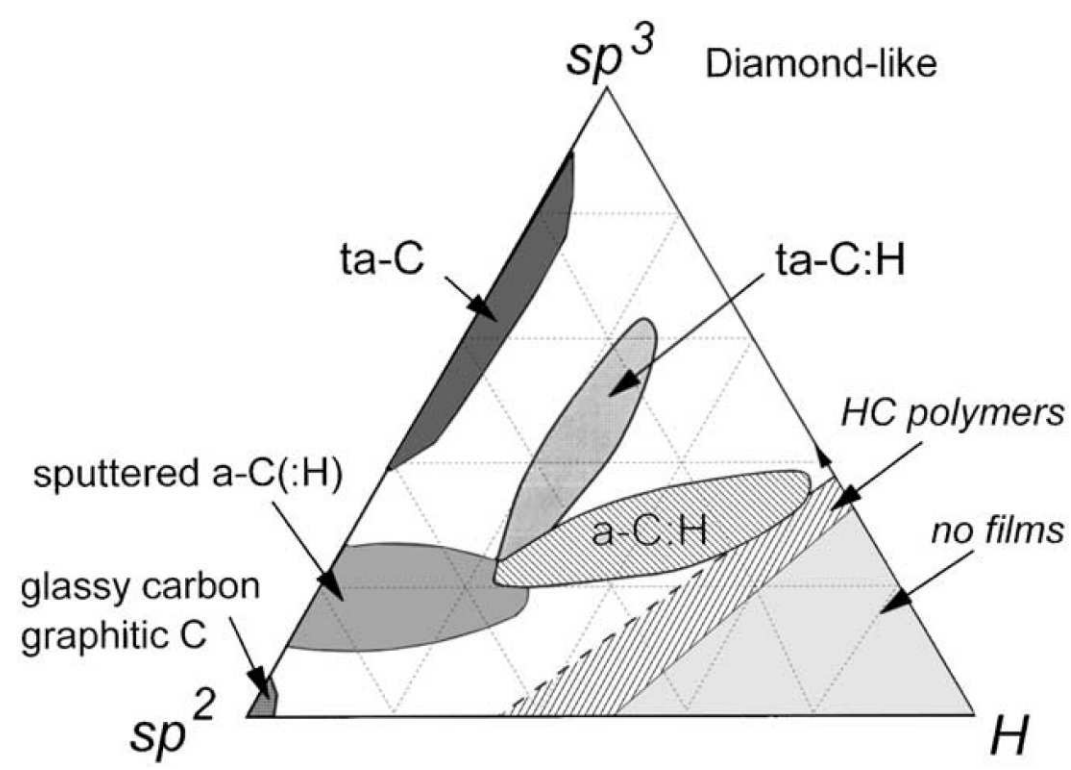

Fig. 1 Phase diagram of amorphous carbon based on pure $\mathrm{C}$ and $\mathrm{H}$ compounds (from Robertson 2002).

elements can also be incorporated in the ring system as well as in the side groups.

By introducing $\mathrm{H}$ into graphite $\left(s p^{2}\right)$ and diamond $\left(s p^{3}\right)$, a variety of amorphous $\mathrm{C}-\mathrm{H}$ alloys can be created. Geometric structures of different long- and short-range can be created by varying the $s p^{2} / s p^{3}$ hybridization ratios. A schematic of possible structures of amorphous carbonaceous solids is shown in Figure 1. The lower left corner of the triangle represents graphite, the top corner represents diamonds, PAHs are on the bottom edge, and various forms of amorphous hydrogenated carbon can exist in the middle. The infrared spectra of these amorphous carbonaceous materials (Dischler et al. 1983) resemble the astronomical UIE bands seen in planetary nebulae and pre-planetary nebulae (Figure 2). Since these amorphous carbonaceous solids have absorption bands in the visible, they can be easily excited by visible light from stars.

Carbonaceous solids with the structures described in Figure 1 have also been artificially synthesized in the laboratory. The techniques that have been used include microwave irradiation of plasma of 4-torr methane (Sakata et al. 1987; Godard et al. 2011), hydrocarbon flame or arc-discharge in a neutral of hydrogenated atmosphere (Colangeli et al. 1997; Mennella et al. 2003), laser ablation of graphite in a hydrogen atmosphere (Scott and Duley 1996; Mennella et al. 1999; Jäger et al. 2008), infrared laser pyrolysis of gas phase molecules $\left(\mathrm{C}_{2} \mathrm{H}_{4}, \mathrm{C}_{4} \mathrm{H}_{6}\right)$ into C-based nanoparticles (Herlin et al. 1998), photolysis of methane at low temperatures (Dartois et al. 2004), and flame combustion of $\mathrm{C}_{2} \mathrm{H}_{2}, \mathrm{C}_{2} \mathrm{H}_{4}, \mathrm{C}_{3} \mathrm{H}_{6}$ mixed with $\mathrm{O}_{2}$ into soot (Pino et al. 2008; 


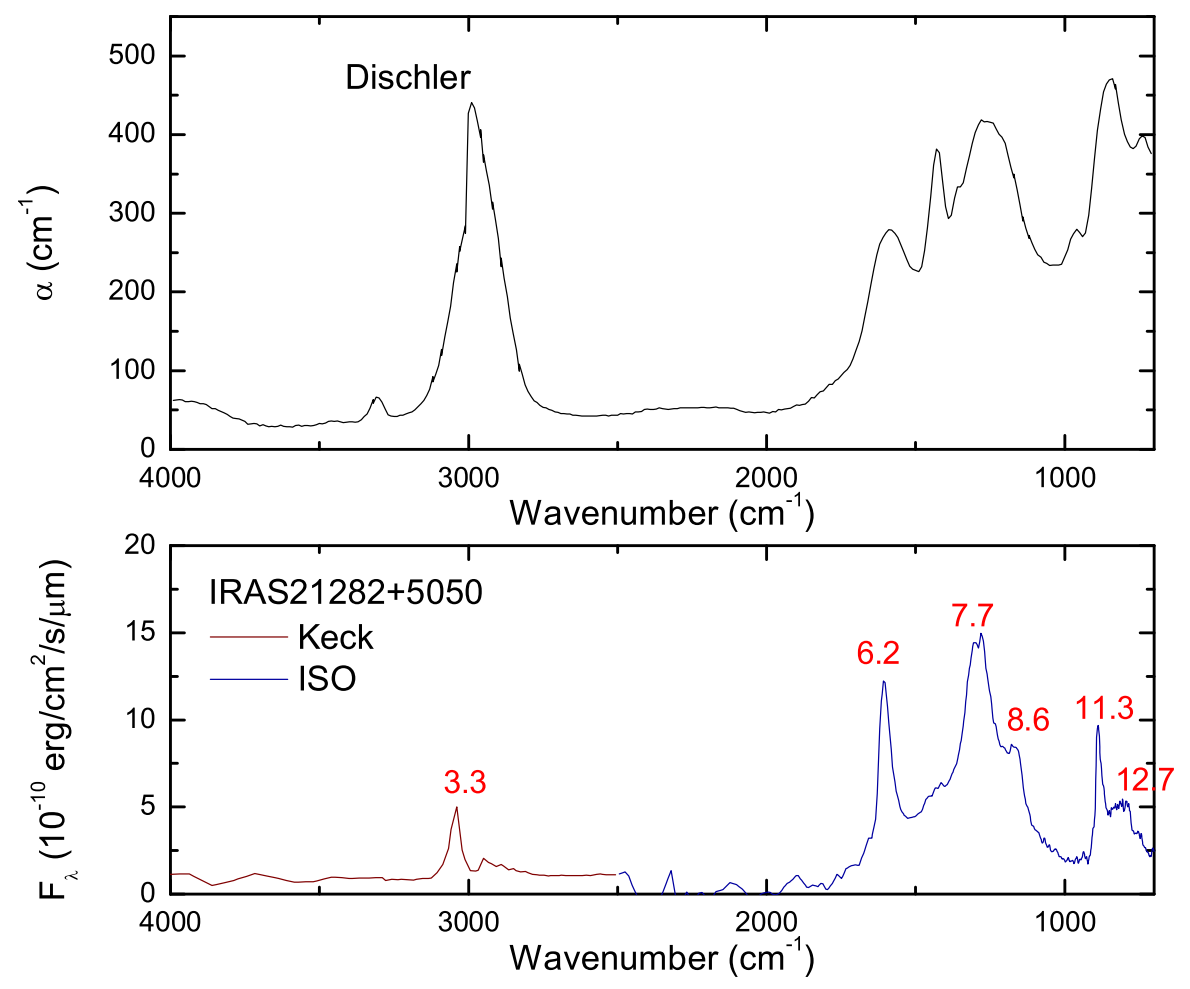

Fig. 2 Laboratory infrared spectra of hydrogenated amorphous carbon (top, from reference (Dischler et al. 1983)) compared to the astronomical spectrum of the planetary nebula IRAS $21282+5050$ (bottom panel). The UIE bands are labeled by their wavelengths in $\mu \mathrm{m}$.

Carpentier et al. 2012). The ease that these compounds can be created suggests that complex amorphous organic solids may be widely present in space.

\section{Fullerenes and MAONs}

The fullerene $\left(\mathrm{C}_{60}\right)$ molecule was first synthesized in the laboratory and later found in carbonaceous rocks (Buseck 1992). Geological fullerenes are believed to be either brought to Earth from extraterrestrial impactors, or formed in high temperature conditions during impacts. The first astronomical detection of fullerene was in the H-poor planetary nebula Tc-1 (Cami et al. 2010). It was later also detected in reflection nebulae (Sellgren et al. 2010), in Hrich planetary nebulae (García-Hernández et al. 2010), and in pre-planetary nebulae (Zhang and Kwok 2011). Although Tc-1 does not show UIE bands, the aliphatic 8 and $12 \mu \mathrm{m}$ plateau features are strongly present (Figure 3 ). In the pre-planetary nebula IRAS $01005+7910$, all three plateau features at 8 , 12 , and $17 \mu \mathrm{m}$ seen in planetary nebulae with UIE bands are found (Zhang and Kwok 2013). As a matter of fact, plateau features are present in all 


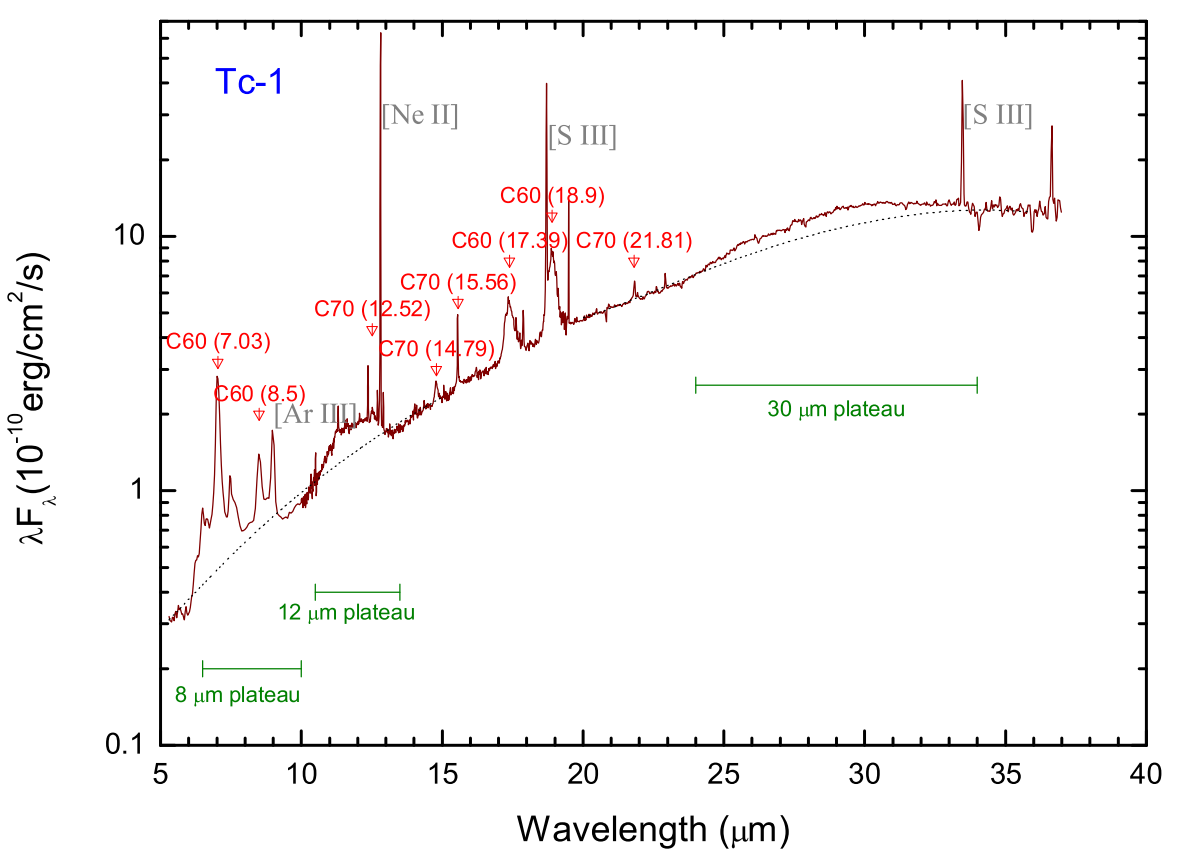

Fig. 3 The Spitzer IRS spectrum of planetary nebula Tc- 1 shows $\mathrm{C}_{60}$ and $\mathrm{C}_{70}$ emission bands (in red) as well as broad emission plateau features at 8,12 , and $30 \mu \mathrm{m}$ (in green). The narrow lines are atomic lines (in grey). No UIE bands are seen in this object.

planetary nebulae with $\mathrm{C}_{60}$ (Otsuka et al. 2013). Several $\mathrm{C}_{60}-\mathrm{PN}$ (Tc-1, SMP SMC 16, SMP LMC 56) have plateau features but no UIE bands, implying the carriers of the plateau features are not aromatic in origin. The possibility that MAONs are precursors of fullerenes has been speculated (García-Hernández et al. 2012; Bernard-Salas et al. 2012).

\section{Organics in the Solar System}

It is well known that carbonaceous meteorites contain insoluble organic matter (IOM) that is similar in chemical structure to kerogen (Kerridge 1999). IOM is composed of highly substituted single ring aromatics, substituted furan/pyran moieties, highly branched oxygenated aliphatics, and carbonyl groups (Cody et al. 2011) and contains other hetero-elements such as S and N (Derenne and Robert 2010). Average elemental abundance of IOM can be represented by the formula $\mathrm{C}_{100} \mathrm{H}_{46} \mathrm{~N}_{10} \mathrm{O}_{15} \mathrm{~S}_{4.5}$ (Pizzarello and Shock 2010). At the very least, this is evidence that IOM is a product of abiotic organic chemistry and Nature can make complex organics without life.

In planetary satellites, we have learned from the Cassini results that organic haze is a major component in the atmosphere of Titan and these nanoparticles are blown into dunes by Titan's surface winds. The surface of Titan is 
covered by lakes of liquid methane and ethane and the total amount of hydrocarbons on Titan is larger than the oil and gas reserves on Earth (Lorenz et al. 2008). There has been some debate as to whether these complex organics are tholins (Sagan and Khare 1979) or HCN polymers (Matthews and Minard 2008). Comparisons between the $3.4 \mu \mathrm{m}$ feature observed in Titan haze is similar to that seen in comets and pre-planetary nebulae (Kim et al. 2011). It has been suggested that the carrier of the $3.4 \mu \mathrm{m}$ feature in comets originates from interstellar organic matter embedded in comets during the early stages of the Solar System (Mennella 2010).

In the past, it was commonly believed that planets and other Solar System objects formed from a well-mixed primordial nebula of chemically and isotopically uniform composition. All solid bodies were assumed to be made afresh and no solid primordial material was thought to have survived the formation process (Suess 1965). Isotopic studies of meteorites have identified solid grains of presolar origin, including diamonds, $\mathrm{SiC}$, corundum, and spinel. These presolar grains are consistent with their being of AGB origin (Davis 2011). The amount of IOM brought in during early heavy bombardment is estimated to account for 1-3\% in weight contribution to the terrestrial organic pool (Mautner et al. 1995). It is therefore quite possible that the early Earth was enriched by extraterrestrial organics.

\section{Implications on the Origin of Life}

Through the technique of infrared spectroscopy, we now know that old stars are able to synthesize complex organic solids with aromatic/aliphatic structures over very short $\left(\sim 10^{3} \mathrm{yr}\right)$ time scales under low-density conditions. Since almost all stars go through the AGB-pre-planetary nebulae-planetary nebulae stage, large amount of organics are produced and distributed all over the Galaxy (Kwok 2004). The detection of pre-solar grains in meteorites suggests that grains from AGB stars can survive the journey through the interstellar medium and reach the Solar System. It is probable that stellar organics was part of the primordial materials that formed the Solar System. Macromolecular organics in meteorites, interplanetary dust particles, comets, and planetary satellites show similarities with organics produced by planetary nebulae. Is this a coincidence or do they share a common origin? It would be worthwhile for us to further explore to what extent the Solar System was enriched by stellar organics and to what extent the early Earth was chemically enriched by extraterrestrial organics. Since planetary systems are now known to be common among stars, the wide production of organic materials by stars in the Galaxy implies that the Earth may not be unique in having been enriched by stellar organics. This may have significant implications on the existence and distribution of life in our Galaxy.

Acknowledgements I thank Kenji Ikehara and the LOC for a well-organized conference and Sandra Pizzarello and the SOC for a stimulating scientific program in Origins 2014. I am 
grateful to my colleagues Yong Zhang and SeyedAbdolreza Sadjadi for their contributions and discussions. This work was partially supported by the Research Grants Council of the Hong Kong Special Administrative Region, China (project no. 17302214).

\section{References}

Allamandola LJ, Tielens AGGM, Barker JR (1985) Polycyclic aromatic hydrocarbons and the unidentified infrared emission bands - Auto exhaust along the Milky Way. Astrophys J, 290, L25-L28

Bernard-Salas J, Cami J, Peeters E, Jones AP, Micelotta ER, Groenewegen MAT (2012). On the Excitation and Formation of Circumstellar Fullerenes. Astrophys J, 757, 41

Buseck PR, Tsipursky SJ, Hettich R (1992) Fullerenes from the geological environment. Science 257:215-217

Cami J, Bernard-Salas J, Peeters E, Malek SE (2010) Detection of $\mathrm{C}_{60}$ and $\mathrm{C}_{70}$ in a Young Planetary Nebula. Science 329:1180

Carpentier Y, Féraud G, Dartois E, Brunetto R, Charon E, Cao A-T, d'Hendecourt L, Bréchignac P, Rouzaud J-N, Pino T (2012). Nanostructuration of carbonaceous dust as seen through the positions of the 6.2 and $7.7 \mu \mathrm{m}$ AIBs. Astron Astrophys, 548, 40

Cataldo F, Keheyan Y, Heymann D (2004) Complex Organic Matter in Space: About the Chemical Composition of Carriers of the Unidentified Infrared Bands (UIBs) and Protoplanetary Emission Spectra Recorded from Certain Astrophysical Objects. Origins of Life and Evolution of the Biosphere, 34, 13-24

Cernicharo J, Agúndez M, Guélin M, Bachiller R (2011) Spectral Line Surveys of Evolved Stars, in IAU Symposium 280, p. 237

Chung S-H, Violi A (2011) Peri-condensed aromatics with aliphatic chains as key intermediates for the nucleation of aromatic hydrocarbons. Proceedings of the Combustion Institute, 33(1), 693-700

Cody GD, Heying E, Alexander CMO, Nittler LR, Kilcoyne ALD, Sandford SA, Stroud RM (2011) Establishing a molecular relationship between chrondritic and cometary organic solids. Proc Natl Acad Sci USA 108 (48):1917119176

Colangeli L, Bussoletti E, Pestellini CC, Mennella V, Palomba E, Palumbo P, Rotundi A (1997). Laboratory simulation of carbon compounds expected in different astrophysical environments. Advances in Space Research, 20, $1617-1627$

Dartois E (2011) Observations of Interstellar Carbon Compounds. EAS Publications Series. 46, 381

Dartois E et al (2007) IRAS 08572+3915: constraining the aromatic versus aliphatic content of interstellar HACs. Astron Astrophys, 463, 635-640

Dartois E, Muoz Caro GM, Deboffle D, d'Hendecourt L (2004). Diffuse interstellar medium organic polymers. Photoproduction of the 3.4, 6.85 and 7.25 $\mathrm{m}$ features. Astron Astrophys, 423, L33-L36 
Davis, A. M. (2011). Cosmochemistry Special Feature: Stardust in meteorites. Proceedings of the National Academy of Science, 108, 19142-19146

Derenne S, Robert F (2010) Model of molecular structure of the insoluble organic matter isolated from Murchison meteorite. Meteoritics and Planetary Science 45:1461-1475

Dischler B, Bubenzer A, Koidl P (1983) Bonding in hydrogenated hard carbon studied by optical spectroscopy. Solid State Communications, 48(2), 105-108

Duley WW, Williams DA (1981) The infrared spectrum of interstellar dust Surface functional groups on carbon. Monthly Notices of the Royal Astronomical Society, 196, 269-274

Duley WW, Williams DA (1979). Are there organic grains in the interstellar medium. Nature, 277, 40

García-Hernández DA, Manchado A, Garca-Lario P, Stanghellini L, Villaver E, Shaw RA, Szczerba R, Perea-Caldern JV (2010) Formation of Fullerenes in H-containing Planetary Nebulae. Astrophys J 724:L39-L43

García-Hernández DA, Villaver E, García-Lario P, Acosta-Pulido JA, Manchado A, Stanghellini L, Shaw RA, Cataldo F (2012). Infrared Study of Fullerene Planetary Nebulae. Astrophys J, 760, 107.

Godard M et al (2011) Ion irradiation of carbonaceous interstellar analogues. Effects of cosmic rays on the $3.4 \mathrm{~m}$ interstellar absorption band. Astron Astrophys, 529, 146

Herlin N, Bohn I, Reynaud C, Cauchetier M, Galvez A, Rouzaud J-N (1998). Nanoparticles produced by Laser Pyrolysis of hydrocarbons: analogy with carbon cosmic dust. Astron Astrophys, 330, 1127-1135

Jäger C, Mutschke H, Henning T, Huisken F (2008). Spectral Properties Of Gas-Phase Condensed Fullerene-Like Carbon Nanoparticles From FarUltraviolet To Infrared Wavelengths. Astrophys J, 689(1), 249-259

Jones AP, Duley WW, Williams DA (1990) The structure and evolution of hydrogenated amorphous carbon grains and mantles in the interstellar medium. Quarterly Journal of the Royal Astronomical Society, 31, 567-582.

Kerridge JF (1999) Formation and Processing of Organics in the Early Solar System. Space Sci Rev 90:275-288

Kim SJ, Jung A, Sim CK, Courtin R, Bellucci A, Sicardy B, Song IO, Minh YC (2011) Retrieval and tentative indentification of the $3 \mathrm{~m}$ spectral feature in Titan's haze. Planet Space Sci 59:699-704

Knacke RF (1977). Carbonaceous compounds in interstellar dust. Nature, 269, $132-134$

Kwok S (1993) Proto-planetary nebulae. Ann Rev Astron Astrophys, 31, 63-92

Kwok S (2000) The Origin and Evolution of Planetary Nebulae, CUP

Kwok S (2004). The synthesis of organic and inorganic compounds in evolved stars. Nature, 430, 985-991.

Kwok S, Volk K, Bernath P (2001) On the Origin of Infrared Plateau Features in Proto-Planetary Nebulae. Astrophys J, 554, L87-L90

Kwok S, Volk K, Bidelman WP (1997). Classification and Identification of IRAS Sources with Low-Resolution Spectra. Astrophys J Suppl, 112, 557 
Kwok S, Volk K, Hrivnak BJ (1999) Chemical evolution of carbonaceous materials in the last stages of stellar evolution. Astron Astrophys, 350, L35-L38.

Kwok S, Zhang Y (2011) Mixed aromatic-aliphatic organic nanoparticles as carriers of unidentified infrared emission features. Nature, 479, 80-83

Kwok S, Zhang Y (2013) Unidentified Infrared Emission Bands: PAHs or MAONs? Astrophys J, 771, 5

Lorenz RD et al (2008) Titan's inventory of organic surface materials. Geophys Res Lett 35:02206

Matthews CN, Minard RD (2008) Hydrogen cyanide polymers connect cosmochemistry and biochemistry. In IAU Symposium 251: Organic Matter in Space, eds. S. Kwok \& S. Sandford, CUP, p. 453-457

Mautner MN, Leonard RL, Deamer DW (1995) Meteorite organics in planetary environments: hydrothermal release, surface activity, and microbial utilization. Planet Space Sci 43:139-147

Mennella V (2010) H Atom Irradiation of Carbon Grains under Simulated Dense Interstellar Medium Conditions: The Evolution of Organics from Diffuse Interstellar Clouds to the Solar System. Astrophys J 718:867-875

Mennella V, Baratta GA, Esposito A, Ferini G, Pendleton YJ (2003). The Effects of Ion Irradiation on the Evolution of the Carrier of the 3.4 Micron Interstellar Absorption Band. Astrophys J, 587, 727-738.

Mennella V, Brucato JR, Colangeli L, Palumbo P (1999) Activation of the 3.4 Micron Band in Carbon Grains by Exposure to Atomic Hydrogen. Astrophys J, 524, L71-L74

Otsuka M, Kemper F, Hyung S, Sargent BA, Meixner M, Tajitsu A, Yanagisawa $\mathrm{K}$ (2013) The Detection of $\mathrm{C}_{60}$ in the Well-characterized Planetary Nebula M1-11. Astrophys J 764:77

Papoular R (2001) The use of kerogen data in understanding the properties and evolution of interstellar carbonaceous dust. Astron Astrophys, 378, 597607

Papoular R, Conrad J, Giuliano M, Kister J, Mille G (1989) A coal model for the carriers of the unidentified IR bands. Astron Astrophys, 217, 204-208

Pendleton YJ, Sandford SA, Allamandola LJ, Tielens AGGM, Sellgren K (1994) Near-infrared absorption spectroscopy of interstellar hydrocarbon grains. Astrophys J, 437, 683-696

Pino T, Dartois E, Cao A-T, Carpentier Y, Chamaill3́ T, Vasquez R, Jones AP, D'Hendecourt L, Bréchignac P (2008) The $6.2 \mu \mathrm{m}$ band position in laboratory and astrophysical spectra: a tracer of the aliphatic to aromatic evolution of interstellar carbonaceous dust. Astron Astrophys, 490, 665-672

Pizzarello S, Shock E (2010) The Organic Composition of Carbonaceous Meteorites: The Evolutionary Story Ahead of Biochemistry. Cold Spring Harbor Perspectives in Biology 2 (3)

Puetter RC, Russell RW, Willner SP, Soifer BT (1979) Spectrophotometry of compact H II regions from 4 to 8 microns. Astrophys J, 228, 118-122

Robertson J (2002) Diamond-like amorphous carbon. Materials Science and Engineering: R: Reports, 37(46), 129-281 
Russell RW, Soifer BT, Willner SP (1977). The 4 to 8 micron spectrum of NGC 7027. Astrophys J, 217, L149-L153.

Sagan C, Khare BN (1979) Tholins - Organic chemistry of interstellar grains and gas. Nature 277:102-107

Sakata A, Wada S, Onaka T, Tokunaga AT (1987) Infrared spectrum of quenched carbonaceous composite (QCC). II - A new identification of the 7.7 and 8.6 micron unidentified infrared emission bands. Astrophys J, 320, L63-L67

Scott A, Duley WW (1996). The Decomposition of Hydrogenated Amorphous Carbon: A Connection with Polycyclic Aromatic Hydrocarbon Molecules. Astrophys J, 472, L123.

Sellgren K, Werner MW, Ingalls JG, Smith JDT, Carleton TM, Joblin C (2010) C60 in Reflection Nebulae. Astrophys J Letters 722:L54-L57

Smith JDT et al (2007) The Mid-Infrared Spectrum of Star-forming Galaxies: Global Properties of Polycyclic Aromatic Hydrocarbon Emission. Astrophys J, 656, 770-791

Spoon HWW et al (2004) Fire and ice: Spitzer Infrared Spectrograph (IRS) mid-infrared spectroscopy of IRAS F00183-7111. Astrophys J Supplement Series, 154(1), 184-187

Suess HE (1965) Chemical Evidence Bearing on the Origin of the Solar System. Annu Rev Astron Astrophys 3:217

Wickramasinghe DT, Allen DA (1980) The 3.4-micron interstellar absorption feature. Nature, 287, 518

Yamagishi M, Kaneda H, Ishihara D, Kondo T, Onaka T, Suzuki T, Minh YC (2012) AKARI near-infrared spectroscopy of the aromatic and aliphatic hydrocarbon emission features in the galactic superwind of M 82. Astron Astrophys, 541, 10

Zhang Y, Kwok S (2011) Detection of $\mathrm{C}_{60}$ in the Protoplanetary Nebula IRAS 01005+7910. Astrophys J 730:126

Zhang Y, Kwok S (2013) On the detections of $\mathrm{C}_{60}$ and derivatives in circumstellar environments. Earth, Planets, and Space 65:1069-1081

Ziurys L (2014) Prebiotic Chemical Evolution in the Astrophysical Context, presented at Origins 2014, Nara, Japan 